\title{
SARS-CoV-2 en América Latina y Caribe: Las tres encrucijadas para el pensamiento crítico en salud
}

\author{
SARS-CoV-2 in Latin America and the Caribbean: \\ The three intersections for critical thinking in health
}

${ }^{1}$ Facultad Latinoamericana de Ciencias Sociales República Dominicana, Programa de Salud Internacional. GT Salud Internacional del Consejo Latinoamericano de Ciencias Sociales. Estados Unidos 1168, C1101AAX. Ciudad de Buenos Aires Argentina. gbasile@flacso.org.do

\begin{abstract}
Thinking about the SARS-CoV-2 pandemic implies the study of general and unique dimensions for the historical evolution of Latin America and the Caribbean. From the individual to the collective, from biomedical sciences to social sciences and collective health, from risk groups to exclusive societies and the inequities constituting the colonial, patriarchal, modern capitalist heritage in the State and societies. The objective of this article is to review what are called the three intersections for Latin American critical health thinking. Seeking to analyze and reflect on the assumptions and logic present in the responses to the health emergency with reference to: 1 . Critical health theory and its intersections with Latin American critical thinking; 2. The decolonial implications of problematizing the State and public health systems; and 3. The geopolitics of global health security as a roadmap for the global North. They outline approaches on the risks of capitalism's acceleration of the post-pandemic disaster and the alternative ways of addressing creative tensions in the reconstruction of emancipatory processes for regional health sovereignty and Health from the South.

Key words SARS-CoV-2, Global Health Security, Latin American critical thinking, South South International Health, Sanitary Sovereignty
\end{abstract}

Resumen Pensar la pandemia de SARS-CoV-2 implica el estudio de dimensiones generales y singulares para el devenir histórico de América Latina y Caribe. De lo individual a lo colectivo, de las ciencias biomédicas a las ciencias sociales y la salud colectiva, de los grupos de riesgos a las sociedades excluyentes y las inequidades constitutivas de la herencia colonial, patriarcal, capitalista moderna en el Estado y las sociedades. El objetivo de este artículo es revisar lo que se denomina las tres encrucijadas para el pensamiento crítico latinoamericano en salud. Buscando analizar y reflexionar sobre los presupuestos y lógicas presentes en la respuesta a la emergencia sanitaria en referencia a: 1. La teoría crítica en salud y sus intersecciones con el pensamiento crítico latinoamericano; 2. las implicancias decoloniales de problematizar el Estado y los sistemas de salud pública, y 3. la geopolítica de la seguridad sanitaria global como hoja de ruta del Norte global. Se esbozan aproximaciones en los riesgos de aceleración del capitalismo del desastre post-pandemia y los caminos alternativos de abordaje de las tensiones creativas en la reconstrucción de procesos emancipatorios para la soberanía sanitaria regional y una Salud desde el Sur. Palabras claves SARS-CoV-2, Seguridad Sanitaria Global, Pensamiento crítico latinoamericano, Salud Internacional Sur Sur, Soberanía Sanitaria 


\section{Introducción}

Desde la confirmación del primer caso de SARSCoV-2 en América Latina y Caribe, se observa un umbral epidemiológico indefinido de casos ascendente para la región, conjuntamente con una curva ascendente de pánico colectivo, desinformación/sobreinformación, naturalización de la desprotección en sociedades excluyentes, radicalización de las desigualdades e inequidades por género, etnia y clases sociales, cierta invisibilidad de las fragilidades estructurantes de los sistemas de salud pública y una toma de decisiones basadas en doctrina del shock hacia la sociedad ${ }^{1}$.

La premisa del escritor uruguayo Mario Benedetti de "cuando teníamos todas las respuestas, nos cambiaron las preguntas", sea quizás necesaria como objetivo fundamental para el pensamiento crítico latinoamericano en salud. Es un momento de novedad, donde pareciera útil revisar los lentes epistémicos con que se creía conocer el mundo y el campo de la salud, es decir, repensar la epistemología con que se observa, estudia y analiza la pandemia. Revitalizar la teoría crítica en salud, implica sobre todo en el contexto particular recuperar la capacidad de dudar, preguntar(se) y asumir con humildad el conocimiento como campo de lo complejo, contingente y multidimensional ${ }^{2}$.

El objetivo del presente artículo es revisar las tres encrucijadas para el pensamiento crítico latinoamericano en salud. Buscando analizar y reflexionar sobre lógicas que están presentes en cada una de estas en el escenario regional de respuesta al riesgo epidémico de SARS-CoV-2. Entendiendo y definiendo encrucijadas (Diccionario Real Academia Española: https://dle.rae.es/ encrucijada) con sus tres posibles acepciones.

\section{Primera encrucijada: la parálisis de la ciencia crítica en salud}

Los sustentos ideológicos, concepciones y acciones técnico - políticas y su proyección sobre la sociedad que expresaron la ciencia biomédica clínica individual y la enfermología pública ${ }^{3}$ actualmente en el marco del SARS-CoV-2 generaron un complejo espiral de patologización, conductismo individual, darwinismo social, (neo) higienismo y transferencia de protocolización de control de infecciones hospitalarias hacia la sociedad como estrategia de afrontamiento a la pandemia. Con una toma de decisiones públicas basadas en la predicción e inferencia conjugadas en diversos países con militarización creciente en el marco de abordajes sectorializados y neoliberalizados del cuidado de la salud colectiva ${ }^{4}$.

Ahora bien, en este contexto el saber (bio) médico como modelo epistemológico hegemónico ${ }^{5}$ rápidamente excedió la explicación y el estudio del mundo de la enfermedad del Covid-19, y se ofreció como pertinente para producir conocimiento acerca de la salud y la calidad de vida de la sociedad.

Ante la terapia de shock, la ciencia crítica latinoamericana en salud por momentos sufrió cierta parálisis expectante o peor aún fue colonizada por la secularización biomédica extrema con verdades únicas, repetibles, totalizantes que incluso el pensamiento médico-social desarrollista tendió a reproducir.

El arsenal teórico con que actualmente se piensa y problematiza la pandemia podría estar obsoleto, falto de creatividad e innovación científica emancipatoria.

Las posiciones más disruptivas, rebeldes, rupturistas las encarnan los pensamientos más reaccionarios en las sociedades. Boaventura de Sousa Santos ${ }^{6}$ lo llama la pérdida de sustantivos. Durante mucho tiempo la teoría crítica tuvo palabras que sólo usaban las y los teóricos críticos, las y los pensadores de las alternativas. Hoy sólo queda la adjetivación a la teoría convencional. El Norte global habla de democracia, y se agrega democracia "participativa", "deliberativa"; si la teoría convencional habla de cuarentenas, se habla de cuarentenas "preventivas" o "sociales". Y la lista continúa.

Recuperar el pensamiento crítico en salud expresado en la fructífera trayectoria de la medicina social y salud colectiva latinoamericana y caribeña ${ }^{7}$ pareciera ser esencial para volver a traer en el medio del tránsito de la emergencia y post-emergencia sanitaria la preocupación por observar no solamente la enfermedad y la muerte sino la necesidad de reflexionar, comprender y actuar sobre la salud de y con la sociedad, sin descuidar las primeras. Más que ver sólo objetos de intervención individual y control de poblaciones, el intento por mirar sujetos y relaciones sociales que impactan en estilos, modos y patrones de vida y procesos de salud-enfermedad-atención. Revisar la confianza quizás ciega en una salud pública funcionalista que nuevamente ubicó en la tecnología positivista manejada por el Estado como infalible y única para controlar y prevenir los riesgos y la enfermedad a nivel colectivo. Como lo expresa Edmundo Granda: "cuidar la salud del Estado y de la ciencia-técnica, actuando sobre el riesgo de enfermar de la población a su 
cargo; debe observar a la población, pero a través de los cristales de la norma estatal y de la razón instrumental; e intervenir sobre la población transformada en objeto"3.

El devenir de lo general, particular y singular en las determinaciones sociales de la salud y los modos de vivir, enfermar y morir ${ }^{8}$, nos desafía a un estudio de la gravedad de una enfermedad epidémica en un contexto particular, en un momento particular, en una población particular, pero entrelazado a un sistema mundo que se considera totalizante y universal incluso en el campo de la salud ${ }^{4}$.

La conexión entre lo natural, lo biológico y lo social como proceso determinante de la emergencia de Covid-19 a partir del metabolismo Sociedad-Naturaleza ${ }^{9}$. Los cambios en las dinámicas poblacionales y de urbanización malsana y ecológicamente insustentables, la pérdida del equilibrio natural de los ecosistemas, la introducción de especies exóticas, tráfico ilegal o caza, en definitiva, la brutal y acelerada depredación de la sociedad sobre la naturaleza es quizás la mayor amenaza hoy a la salud colectiva.

Las implicancias de refundar una praxis de encuentro y complementariedad entre el pensamiento crítico latinoamericano ${ }^{10}$ y la salud colectiva/medicina social pudiera ser un camino para responder a esta encrucijada. Esto significa asumir tensiones creativas transdisciplinares $\mathrm{e}$ intergeneracionales.

\section{Segunda encrucijada: los Sistemas de Salud y el Estado}

En general la dimensión del Estado en la región tomó cuerpo e institucionalización a través de cuarentenas, (neo)higienismo individual y policía médica en el siglo XXI con cierto asistencialismo focalizado ${ }^{4}$. Esto fue conjugado con acelerados arreglos institucionales en lógica de preparación (camas, ventilación mecánica, otros) para una respuesta en sistemas de salud pública crónicamente desfinanciados, fragilizados, estratificados, segmentados, financierizados y especialmente con una creciente pérdida de capacidades de cuidado y protección de la salud de las sociedades ${ }^{11}$.

El Estado fue reducido a la preparación de un aparato burocrático externo a la sociedad en la dinámica de afrontamiento al Covid-19. El Estado latinoamericano y caribeño pareciera tener en su genética de sus modos de actuar una selectividad estratégica, siéndole más fácil solventar y responder unos problemas que a otros ${ }^{12}$. El pasado, condensado en las instituciones, pesa en el presente. El Estado es una condensación institucional de las relaciones sociales y de poder al interior de una sociedad. Es evidente que los Estados arrastran el peso de su pasado: la path dependence, esto es, la dependencia de la trayectoria. A decir de Foucault ${ }^{13}$ la gubernamentalidad en salud y las prácticas que construyen estatalidad sanitaria que vienen de la trayectoria colonial de la doctrina panamericana ${ }^{14}$.

La conjugación de comités operativos de emergencias de salud pública atestados de expertos clínicos en su mayoría varones, burocracias estatales con fuerzas de seguridad y/o militares en el centro de las instituciones públicas, $y$, por otra parte, sin espacios de simetría de poder con referentes populares, de mujeres, de indígenas y afrodescendientes, de trabajadores organizados muestra una materialidad selectiva. Esto habla en cierta medida de la selectividad estratégica del Estado a la hora de gestionar la pandemia. Hay cosas que le salen más fácil al Estado latinoamericano y caribeño. Para algunos militarizar, vigilar, castigar. Para otros desproteger los bienes públicos y comunes para la vida. La estatalidad segregada que trae una herencia de colonialidad moderna, capitalista periférica y patriarcal ${ }^{15}$.

Rever la arquitectura institucional e intervención del Estado en la sociedad que se tendió a operacionalizar ante el riesgo epidémico de Covid-19. Respuesta que trajo en su genética lo que denominamos panamericanismo sanitario en muchos casos con recetas y enlatados de repetición ${ }^{16}$.

Postulados de estilos de vida hiper higienizados, cambios en comportamientos individuales, que se narran repetidamente e intentan implementarse en sistemas de salud neoliberalizados y sociedades excluyentes, inequitativas y desiguales como las latinoamericanas y caribeñas.

Es evidente que los modelos de protección social eurocéntricos en su patrón liberal residual, de meritocracia bismarkiano o el universalismo beverige no permitieron resolver la cuestión social de la desigualdad en salud en Latinoamérica y Caribe. Incluso porque fueron teorizaciones desarrolladas y basadas en fundamentos del Norte global. Las oleadas de procesos de reformas al Estado y los sistemas de salud comandados por el Banco Mundial, la Organización Panamericana de la Salud (OPS) y el Banco Interamericano de Desarrollo, reconfiguraron los bienes públicos y colectivos y fragilizaron aún más la débil situación de los sistemas sanitarios. La necesidad de descolonizar(nos) de estas teorías y políticas so- 
bre los sistemas de salud latinoamericanos y caribeños en el siglo XXI continúa siendo una deuda pendiente y otra encrucijada regional ${ }^{11}$.

\section{Tercera encrucijada: La seguridad sanitaria como premisa geopolítica del Norte global}

La dimensión geopolítica fue indisimulable desde que la pandemia tuvo un tránsito de extrema alerta y atención pública en gran medida por la grave afectación en los países del Norte global. Otras enfermedades y epidemias (o la misma Covid-19) en el Sur global nunca tuvieron ni hubieran tenido esta centralidad. La doctrina de la salud internacional panamericana y la salud global liberal en general orgánicamente responden a esta geopolítica del poder y conocimiento del Norte global y su sistema mundo contemporáneo acelerado, deshumanizante y depredador de la naturaleza y la vida ${ }^{16}$.

Así la tercera encrucijada es la referida a las implicancias de la geopolítica de la seguridad sanitaria global ${ }^{17}$. Las amenazas para el Norte global en la circulación de enfermedades infecciosas y patógenos implica obligar a los Estados del sur a tomar una serie de medidas sanitarias que responden más a las vulnerabilidades de los Estados occidentales que a las necesidades reales de salud colectiva de las sociedades del Sur global ${ }^{18}$.

Este nexo entre seguridad y salud se convirtió en una narrativa dominante dentro del campo de la salud y lo internacional en las últimas dos décadas. Flor ${ }^{17}$ presenta la perennización del concepto de seguridad sanitaria global como el producto de un proceso que denomina biomedicalización de las relaciones internacionales.

Actualmente este proceso de medicalización de la seguridad global se expande por diferentes vías como son las nuevas diplomacias sanitarias liberales de la autoridad médica internacional y de agentes extra-sanitarios (OCDE, Banco Mundial, corporaciones y complejo industrial farmacéutico, ONGN, otros), el sistema internacional de cooperación al desarrollo Centro-Norte y el filantrocapitalismo sanitario, las intervenciones humanitarias y militares en salud ${ }^{16}$.

La expansión post-Guerra fría de la globalización anclada en el complejo político liberal de fines del siglo XX y continuada en el siglo XXI implicó el punto de partida de esta tesis de seguridad global en salud como interpretación que algunos ubican sus orígenes en el año 1989, cuando el virólogo estadounidense Stephen Morse presentó el término de enfermedad infecciosa emergente (Emerging Infectious Diseases o EID).
La normalización de la securitización sanitaria global y regional resulta clave para comprender el nuevo nexo entre seguridad y salud, asumiendo que demasiados problemas de salud colectiva (además de Covid-19) estarán enmarcados dentro de la narrativa de seguridad sanitaria conjugados con militarización como ya venía ocurriendo con zika, ébola, SARS, gripes y otras epidemias y desastres en América Latina y África ${ }^{19}$. Es importante estudiar las implicaciones de este movimiento expansivo. La "guerra" al Covid-19 y el discurso excepcionalista, pudiera imbricar la lógica de la Escuela de Copenhague, según la cual cualquier problema puede ser percibido como una amenaza a la seguridad: "no necesariamente porque exista una amenaza existencial real sino porque el problema se presenta como una amenaza".

Durante la década de 1990 los expertos en salud y seguridad de los Estados Unidos comienzan a medir la amenaza internacional que suponía la emergencia de nuevos virus desconocidos hasta entonces para su seguridad nacional ${ }^{17}$. El actor que influyó decididamente en considerar que las enfermedades emergentes constituían una amenaza para la seguridad de Estados Unidos fue el Center for Disease Control and Prevention (CDC) argumentando que podían afectar negativamente tanto a sus intereses comerciales geopolíticos como a la salud pública de sus ciudadanos ${ }^{20}$. Sin considerar a las causas estructurantes en el metabolismo sociedad-naturaleza que condicionaban la aparición de estas nuevas enfermedades ${ }^{15}$. Al CDC le seguirán otros centros que producirán programas de securitización en salud como la Johns Hopkins Center for Health Security, Global Health and Security Executive Program Harvard Medical School, Oxford Centre for Tropical Medicine and Global Health, Global Health Security Index, Walter Reed Army Institute of Research, entre otros.

Como señala Flor ${ }^{17}$ las agencias de seguridad y los centros de investigación de Estados Unidos no dirigieron sus esfuerzos a estudiar y transformar los procesos estructurantes de estas enfermedades ni a frenar los procesos de mercantilización de los bienes públicos sino a construir un sistema de vigilancia epidemiológica global que pudiera responder y alertas a las amenazas a "su" seguridad. Así esta narrativa de seguridad sanitaria global fue construyendo un régimen de gobernanza asociado. Entre 1995 y 2005 se crearon las dos principales estrategias que componen este sistema de vigilancia mundial que se lo denominó GOARN (Global Outbreak Alarm Response Network) y las preparaciones pandémicas. Así con esta Red 
Global de Respuesta a Alertas de Brotes, la OMS mantuvo un aparente dominio y éxito en mostrarse como el actor principal para abordar las amenazas de enfermedades para el Norte global y responder a estos intereses geopolíticos ${ }^{21}$. Esto entró en crisis y reingeniería con el Covid-19.

\section{A modo de reflexiones finales}

La especificidad del capitalismo mundialmente periférico de Latinoamérica pone al descubierto riesgos en el proceso de pandemia/post-pandemia en la avidez de los mercados, corporaciones y de las élites por convertir todo desastre y emergencia en lucro ${ }^{1}$. El «capitalismo del desastre», agrega Klein, utiliza el miedo como un efecto dominador sobre la población a quienes el sistema de mercantilización de la vida no les da otra solución más que una promesa de falsa seguridad. Como Klein plantea, el capitalismo necesita de un proceso de destrucción para edificar su consumo y destruirlo nuevamente para volver a construirlo. Aunque el capitalismo global y regional pareciera autoinfligiéndose un daño exorbitante, también puede que este aprovechando la escena para ajustar y perennizar más capitalismo acelerado. Como lo explica la politóloga Wendy Brown ${ }^{22}$, el capitalismo neoliberal es como una termita que deja huellas en la sociedad y cimientos en el Estado capaces de convertir cada necesidad o problema del ser humano en una empresa rentable. El proceso Covid-19 no es la excepción.
Los movimientos en encrucijada entre una biomedicalización líquida y una ciencia crítica en salud paralizada, las acciones de 'paracaídas' donde se bombean recursos a un brote o emergencia de salud pública para sofocar un incendio epidemiológico en particular sin repensar y refundar los Estados y sus sistemas de salud, y la aceleración de la seguridad sanitaria global en un nexo geopolítico entre seguridad y salud plantean nuevos riesgos para la desintegración regional y crecientes niveles de dependencia sanitaria al Norte global como horizonte para el Sur global.

Una vez más esta pandemia demostró la urgente necesidad de reconstruir redes de cooperación, coordinación y toma de decisiones de salud internacional desde una geopolítica del Sur Sur y decolonial produciendo una nueva soberanía sanitaria regional. Revitalizar la integración y autonomía regional en clave emancipatoria de América Latina y Caribe ${ }^{23}$.

Una salud internacional sur sur que no sólo implica esperar el gobierno del Estado para lograr nuevas relaciones intergubernamentales como base para nuevos cimientos de soberanía político-sanitaria. Este camino comienza desde y con las sociedades del Sur global: entre nuestras redes, territorialidades, diálogos interculturales y relaciones transmodernas. Como expresó Rita Segato: "mientras sólo mirábamos el gobierno del Estado, perdimos a la Sociedad". No se trata de una definición, sino de una nueva opción epistémico-político-ética de la necesidad de una Salud desde el Sur. 


\section{Referencias}

1. Klein N. La Doctrina del Shock: el Auge del Capitalismo. Buenos Aires: Paidós Editorial; 2017.

2. Almeida-Filho N. Complejidad y transdisciplinariedad en el campo de la salud colectiva: evaluación de conceptos y aplicaciones. Salud Colectiva 2006; 2(2):123-146.

3. Granda E. A qué llamamos salud colectiva, hoy. Rev Cubana Salud Pública 2004; 30(2):1-20.

4. Basile G. La triada de cuarentenas, neohigienismo y securitización en el SARS-CoV-2: matriz genética de la doctrina del panamericanismo sanitario. Edición Especial Observatorio Social de la Pandemia: CLACSO; 2020.

5. Menéndez E. El modelo médico y la salud de los trabajadores. Salud Colectiva 2005; 1(1):9-32.

6. Santos BS. Introducción: Las Epistemologías del Sur. Buenos Aires: Siglo XXI Editores, CLACSO; 2009.

7. Iriart C, Waitzkin H, Breilh J, Estrada A, Merhy EE. Medicina social latinoamericana: aportes y desafíos. Rev Panamericana Salud Pública 2002; 12(2):128-136.

8. Breilh J. La determinación social de la salud como herramienta de transformación hacia una nueva salud pública (salud colectiva). Rev Facultad Nacional Salud Pública 2013; 31:13-27.

9. Breilh J. Las tres 'S'de la determinación de la vida: 10 tesis hacia una visión crítica de la determinación social de la vida y la salud. In: Nogueira RP, organizador. Determinação Social da Saúde e Reforma Sanitária. Rio de Janeiro: CEBES; 2010. p. 87-125.

10. Altamirano C, Santos BS, Rivas ET, Miró C. Interrogando al pensamiento crítico latinoamericano. Cuadernos Pensamiento Crítico Latinoamericano 2011; 4(43):1-4.

11. Basile G. Repensar y descolonizar las teorías y políticas sobre sistemas de salud en Latinoamérica y Caribe. Cuadernos Pensamiento Crítico Latinoamericano 2020; 73:1-6.

12. Jessop B. The strategic selectivity of the state: reflections on a theme of Poulantzas. J Hellenic Diaspora 1999; 25(1-2):1-37.

13. Foucault M. Seguridad, territorio, población: curso impartido en el Collège de France (1977-1978). São Paulo: Martins Fontes; 2008.
14. Rapoport M. UNASUR, contracara del panamericanismo [Internet]. América Latina en Movimiento; 2008. Disponible en: http://www.amersur.org/Integ/ Rapoport0806.htm

15. Basile G. IV Dossier de SISS. Coronavirus en América Latina y Caribe: entre la terapia de shock de la enfermología pública y la respuesta de la salud colectiva/salud internacional Sur Sur. Buenos Aires: CLACSO; 2020.

16. Basile G. II Dossier de SISS. Salud Internacional Sur Sur: hacia un giro decolonial y epistemológico. Buenos Aires: CLACSO; 2018.

17. Flor JL. La seguridad sanitaria global a debate. Lecciones críticas aprendidas de la 24. ${ }^{\circ}$ EVE. Comillas J Int Relations 2018; 13:49-62.

18. Rushton S. Seguridad de salud global: ¿seguridad para quién? ¿Seguridad de qué? Political Studies 2011; 59(4):779-796.

19. Wenham C, Farias DBL. Securitizing Zika: the case of Brazil. Security Dialogue 2019; 50(5):398-415.

20. King N. Security, disease, commerce: ideologies of postcolonial global health. Social Studies Sci 2002; 32(5-6):763-789.

21. Fidler D. From international sanitary conventions to global health security: the new International Health Regulations. Chinese J Int Law 2005; 4(2):325-392.

22. Brown W. El pueblo sin atributos: La secreta revolución del neoliberalismo. Barcelona: Malpaso Ediciones SL; 2017.

23. Basile G. III Dossier SISS. La Salud del proceso de integración regional: el caso UNASUR Salud ¿soberanía o dependencia sanitaria? Buenos Aires: CLACSO; 2019.

Artículo presentado en 11/06/2020

Aprobado en 03/07/2020

Versión final presentada en 06/07/2020 